\title{
RESONANT NEUMANN EQUATIONS WITH INDEFINITE LINEAR PART
}

\author{
Giuseppina Barletta - Roberto Livrea \\ Nikolaos S. Papageorgiou
}

\begin{abstract}
We consider aseminonlinear Neumann problem driven by the $p$ Laplacian plus an indefinite and unbounded potential. The reaction of the problem is resonant at $\pm \infty$ with respect to the higher parts of the spectrum. Using critical point theory, truncation and perturbation techniques, Morse theory and the reduction method, we prove two multiplicity theorems. One produces three nontrivial smooth solutions and the second four nontrivial smooth solutions.
\end{abstract}

\section{Introduction}

Let $\Omega \subseteq \mathbb{R}^{N}$ be a bounded domain with a $C^{2}$-boundary $\partial \Omega$. In this paper we study the following semilinear Neumann problem

$$
\begin{cases}-\triangle u(z)+\beta(z) u(z)=f(z, u(z)) & \text { in } \Omega \\ \frac{\partial u}{\partial n}=0 & \text { on } \partial \Omega .\end{cases}
$$

Here $\beta \in L^{s}(\Omega), s>N$ and in general is indefinite (i.e. it is sign-changing). Also, $f(z, x)$ is a Carathéodory function (i.e. for all $x \in \mathbb{R}, z \rightarrow f(z, x)$ is measurable and for almost all $z \in \Omega, x \rightarrow f(z, x)$ is continuous) which asymptotically at $\pm \infty$ interacts with the higher parts of the spectrum of $u \rightarrow-\triangle u+\beta u$, $u \in H^{1}(\Omega)$ (resonant equation). Finally by $n(\cdot)$ we denote the outward unit

2010 Mathematics Subject Classification. 35J80, 35J85, 58E05.

Key words and phrases. Resonant equation, critical groups, reduction method, multiple solutions, unique continuation property. 
normal on $\partial \Omega$. The aim of this paper is to prove multiplicity theorems for such resonant problems when the reaction crosses one or more eigenvalues when $x$ moves from 0 to $\pm \infty$ (crossing nonlinearity).

Such problems where studied primarily in the context of Dirichlet equations with no potential $\beta(\cdot)$ (i.e. $\beta \equiv 0$ ) and under stronger regularity conditions on the reaction $f$. First Amann and Zehnder [2] proved that, if the reaction crosses at least an eigenvalue as we move from 0 to $\pm \infty$, then a nontrivial solution exists. Their conditions on $f$ did not allow for resonance to occur. Subsequently, for the same Dirichlet problem there have been some multiplicity theorems by Castro and Lazer [4] (three solutions for nonresonant equations), Chang, Li and Liu [5] (three solutions for nonresonant equations), Liu [12] (four solutions for resonant equations) and Lia nd Zhang [14] (four solutions for resonant equations). As we already mentioned, all the aforementioned works deal with Dirichlet problems with no potential $\beta(\cdot)$ (i.e. $\beta \equiv 0$ ) and under stronger regularity conditions on the reaction $f$. Resonant Neumann equations with $\beta \equiv 0$, were investigated by Filippakis and Papageorgiou [7], Gasinski and Papageorgiou [9], Tang [17] and Tang-Wu [18], using different methods and different hypotheses on the reaction. In fact in [7] resonance at infinity occurs only with respect to the principal eigenvalue $\lambda_{0}=0$ of $\left(-\triangle, H^{1}(\Omega)\right)$, while in [16], [17] resonance occurs only at zero. In [9], the limit as $|x| \rightarrow \infty$ of the quotient $f(z, x) / x$ exists.

Our approach combines variational methods based on the critical point theory together with suitable truncation and perturbation techniques and Morse theory (critical groups). Also,we employ the so-called reduction method, which was first developed by Amann [1], Castro and Lazer [4] and Thews [19] for $C^{2}$ functionals. In the next section, for the convenience of the reader, we recall the main mathematical tools which we will use in this work and also develop the spectral properties of $u \rightarrow-\triangle u+\beta u, u \in H^{1}(\Omega)$.

\section{Mathematical background}

Let $X$ be a Banach space and $X^{*}$ its dual. By $\langle\cdot, \cdot\rangle$ we denote the duality brackets for the pair $\left(X^{*}, X\right)$. Let $\varphi \in C^{1}(X)$. We say that $\varphi$ satisfies the "Cerami condition" (the " $C$-condition" for short), if the following is true:

"Every sequence $\left\{x_{n}\right\}_{n \geq 1} \subseteq X$ such that $\varphi\left(x_{n}\right) \subseteq \mathbb{R}$ is bounded and

$$
\left(1+\left\|x_{n}\right\|\right) \varphi^{\prime}\left(x_{n}\right) \rightarrow 0 \quad \text { in } X^{*} \text { as } n \rightarrow \infty,
$$

admits a strongly convergent subsequence."

This compactness-type condition is in general weaker than the more usual "Palais-Smale condition". Nevertheless it suffices to prove a deformation theorem and from it derive the minimax theory of certain critical values of $\varphi$. One 
such result is the so called "mountain pass theorem" (see, for example, Gasinski and Papageorgiou [8]).

Theorem 2.1. If $\varphi \in C^{1}(X)$ satisfies the $C$-condition, $x_{0}, x_{1} \in X$, $\left\|x_{1}-x_{0}\right\|$ $>r>0$

$$
\max \left\{\varphi\left(x_{0}\right), \varphi\left(x_{1}\right)\right\}<\inf \left[\varphi(x):\left\|x-x_{0}\right\|=r\right]=\eta_{r}
$$

$c=\inf _{\gamma \in \Gamma} \max _{0 \leq t \leq 1} \varphi(\gamma(t))$, where $\Gamma=\left\{\gamma \in C([0,1], X): \gamma(0)=x_{0}, \gamma(1)=x_{1}\right\}$, then $c \geq \eta_{r}$ and $c$ is a critical value of $\varphi$.

Given $\varphi \in C^{1}(X)$ and $c \in \mathbb{R}$, we introduce the following sets:

$$
\begin{aligned}
\varphi^{c} & =\{x \in X: \varphi(x) \leq c\}, \\
K_{\varphi} & =\left\{x \in X: \varphi^{\prime}(x)=0\right\}, \\
K_{\varphi}^{c} & =\left\{x \in K^{\varphi}: \varphi(x)=c\right\} .
\end{aligned}
$$

Also, let $Y_{1}, Y_{2}$ be two topological spaces such that $Y_{2} \subseteq Y_{1} \subseteq X$. For every integer $k \geq 0$, by $H_{k}\left(Y_{1}, Y_{2}\right)$ we denote the $k^{t h}$-relative singular homology group with integer coefficients for the pair $\left(Y_{1}, Y_{2}\right)$. The critical groups of $\varphi \in C^{1}(X)$ at an isolated critical point $x \in X$ with $\varphi(x)=c$ (i.e. $x \in K_{\varphi}^{c}$ ), are defined by

$$
C_{k}(\varphi, x)=H_{k}\left(\varphi^{c} \cap \mathcal{U}, \varphi^{c} \cap \mathcal{U} \backslash\{x\}\right), \quad \text { for all } k \geq 0,
$$

where $\mathcal{U}$ is a neighbourhood of $x$ such that $K_{\varphi} \cap \varphi^{c} \cap \mathcal{U}=\{x\}$. The excision property of singular homology, implies that the above definition of critical groups, is independent of the particular neighbourhood $\mathcal{U}$.

Assume that $\varphi \in C^{1}(X)$ satisfies the C-condition and $-\infty<\inf \varphi\left(K_{\varphi}\right)$. Let $c<\inf \varphi\left(K_{\varphi}\right)$. The critical groups of $\varphi$ at infinity are defined by

$$
C_{k}(\varphi, \infty)=H_{k}\left(X, \varphi^{c}\right) \text { for all } k \geq 0 .
$$

The second deformation theorem (see, for example, Gasinski and Papageorgiou [8, p. 628]), implies that the above definition of critical groups at infinity, is independent of the particular choice of the level $c<\inf \varphi\left(K_{\varphi}\right)$.

Suppose that $K_{\varphi}$ is finite. We set

$$
M(t, x)=\sum_{k \geq 0} \operatorname{rank} C_{k}(\varphi, x) t^{k} \quad \text { for all } t \in \mathbb{R}, \text { all } x \in K_{\varphi}
$$

and

$$
P(t, \infty)=\sum_{k \geq 0} \operatorname{rank} C_{k}(\varphi, \infty) t^{k} \quad \text { for all } t \in \mathbb{R} .
$$

The Morse relation says that

$$
\sum_{x \in K_{\varphi}} M(t, x)=P(t, \infty)+(1+t) Q(t) \quad \text { for all } t \in \mathbb{R},
$$

where $Q(t)=\sum_{k \geq 0} \beta_{k} t^{k}$ is a formal series with nonnegative integer coefficients $\beta_{k}$. In the study of problem (1.1) in addition to the Sobolev space $H^{1}(\Omega)$, 
we will also use the space $C^{1}(\bar{\Omega})$. This is an ordered Banach space with positive cone

$$
C_{+}=\left\{x \in C^{1}(\bar{\Omega}): u(z) \geq 0 \text { for all } z \in \bar{\Omega}\right\} .
$$

This cone has nonempty interior given by

$$
\operatorname{int} C_{+}=\left\{x \in C_{+}: u(z)>0 \text { for all } z \in \bar{\Omega}\right\} .
$$

For $x \in \mathbb{R}$, we set $x^{ \pm}=\max \{0, \pm x\}$ and for $u \in H^{1}(\Omega)$ we define $u^{ \pm}(\cdot)=u(\cdot)^{ \pm}$. We know that

$$
u^{ \pm} \in H^{1}(\Omega), \quad u=u^{+}-u^{-} \quad \text { and } \quad|u|=u^{+}+u^{-} .
$$

In the sequel by $\|\cdot\|$ we denote the norm of $H^{1}(\Omega)$, i.e. $\|u\|=\left(\|D u\|_{2}^{2}+\|u\|_{2}^{2}\right)^{1 / 2}$ for all $u \in H^{1}(\Omega)$. Also, if $h: \Omega \times \mathbb{R} \rightarrow \mathbb{R}$ is a measurable function (for example, a Carathéodory function), then we set

$$
N_{h}(u)(\cdot)=h(\cdot, u(\cdot)) \quad \text { for all } u \in H^{1}(\Omega)
$$

(the Nemytski map corresponding to $h$ ). Finally by $|\cdot|_{N}$ we denote the Lebesgue measure on $\mathbb{R}^{N}$.

Now, let us examine the spectral properties of $u \rightarrow-\triangle u+\beta u, u \in H^{1}(\Omega)$. So, we consider the following linear eigenvalue problem:

$$
-\triangle u(z)+\beta(z) u(z)=\lambda u(z) \quad \text { in } \Omega, \quad \frac{\partial u}{\partial n}=0 \quad \text { on } \partial \Omega .
$$

To analyze problem (2.2) it enough to assume that $\beta \in L^{N / 2}(\Omega)$ when $N \geq 3$ and $\beta \in L^{1}(\Omega)$ for $N=1, \beta \in L^{r}(\Omega)$ with $r>1$ for $N=2$ (by the Sobolev embedding theorem).

In what follows $\sigma: H^{1}(\Omega) \rightarrow \mathbb{R}$ is the $C^{1}$-functional defined by

$$
\sigma(u)=\|D u\|_{2}^{2}+\int_{\Omega} \beta u^{2} d z \quad \text { for all } u \in H^{1}(\Omega) .
$$

Lemma 2.2. If $\beta \in L^{N / 2}(\Omega)$ when $N \geq 3, \beta \in L^{1}(\Omega)$ when $N=1$ and $\beta \in$ $L^{r}(\Omega), r>1$ when $N=2$, then $\widehat{\lambda}_{1}=\inf \left[\sigma(u): u \in H^{1}(\Omega),\|u\|_{2}=1\right]>-\infty$.

Proof. We treat the case $N \geq 3$, the cases $N=1$ and $N=2$ can be handled similarly. We proceed by contradiction. So, suppose that $\widehat{\lambda}_{1}=-\infty$, Then we can find $\left\{u_{n}\right\}_{n \geq 1} \subseteq H^{1}(\Omega)$ such that

$$
\left\|u_{n}\right\|_{2}=1 \quad \text { for all } n \geq 1 \quad \text { and } \quad \sigma\left(u_{n}\right) \rightarrow-\infty \quad \text { as } n \rightarrow \infty .
$$

From (2.3) we see that we can find $n_{0} \geq 1$ such that

$$
\sigma\left(u_{n}\right) \leq-1 \text { for all } n \geq n_{0} .
$$

Suppose that $\left\|u_{n}\right\| \rightarrow \infty$ and let $y_{n}=u_{n} /\left\|u_{n}\right\|, n \geq 1$. Then $\left\|y_{n}\right\|=1$ for all $n \geq 1$ and so we may assume that

$$
y_{n} \rightarrow y \quad \text { in } H^{1}(\Omega) \quad \text { and } \quad y_{n} \rightarrow y \quad \text { in } L^{2}(\Omega) \text { as } n \rightarrow \infty .
$$


Using the Sobolev embedding theorem, we have that $\left\{y_{n}^{2}\right\}_{n \geq 1} \subseteq L^{N /(N-2)}(\Omega)$ is bounded. So, we may assume that

$$
y_{n}^{2} \rightarrow y^{2} \quad \text { in } L^{N /(N-2)}(\Omega), \text { as } n \rightarrow \infty .
$$

Since $\beta \in L^{N / 2}(\Omega)$, we obtain

$$
\int_{\Omega} \beta y_{n}^{2} \rightarrow \int_{\Omega} \beta y^{2}
$$

From (2.4) we have

$$
\sigma\left(y_{n}\right) \leq-\frac{1}{\left\|u_{n}\right\|^{2}} \text { for all } n \geq n_{0},
$$

so (see $(2.5)$ and $(2.6)) \sigma(y) \leq 0$.

Note that $y \neq 0$, or otherwise $y_{n} \rightarrow 0$ in $H^{1}(\Omega)$, which contradicts the fact that $\left\|y_{n}\right\|=1$ for all $n \geq 1$. On the other hand, we have (see (2.3))

$$
\left\|y_{n}\right\|_{2}=1=\frac{\left\|u_{n}\right\|_{2}}{\left\|u_{n}\right\|}=\frac{1}{\left\|u_{n}\right\|} \rightarrow 0,
$$

so $y=0$, a contradiction.

This proves the boundedness of $\left\{u_{n}\right\} \subseteq H^{1}(\Omega)$ and so we may assume that

$$
u_{n} \rightarrow u \quad \text { in } H^{1}(\Omega) \text { and } \quad \int_{\Omega} \beta u_{n}^{2} d z \rightarrow \int_{\Omega} \beta u^{2} d z .
$$

Hence $\sigma(u) \leq \widehat{\lambda}_{1}=-\infty$, a contradiction (since $u \in H^{1}(\Omega)$ ). Therefore $\widehat{\lambda}_{1}>-\infty$

Using Lemma 2.2, we can find $\widehat{\xi}>\max \left\{0,-\widehat{\lambda}_{1}\right\}$ large such that

$$
\sigma(u)+\widehat{\xi}\|u\|_{2}^{2} \geq \widehat{c}\|u\|_{2}^{2} \quad \text { for some } \widehat{c}>0 \text { and all } u \in H^{1}(\Omega) .
$$

We introduce the following equivalent inner product on $H^{1}(\Omega)$,

$$
(u, y)_{*}=\int_{\Omega}(D u, D y)_{\mathbb{R}^{N}} d z+\int_{\Omega}(\beta(z)+\widehat{\xi}) u(z) y(z) d z \quad \text { for all } u, y \in H^{1}(\Omega) .
$$

Given $g \in L^{2}(\Omega)$, by the Riesz representation theorem, we can find a unique $u \in H^{1}(\Omega)$ such that

$$
(u, y)_{*}=\int_{\Omega} g y d z \quad \text { for all } y \in H^{1}(\Omega) .
$$

So, we can define a linear map $K_{*}: L^{2}(\Omega) \rightarrow H^{1}(\Omega)$ by setting $K_{*}(g)=u$. Let $i: H^{1}(\Omega) \rightarrow L^{2}(\Omega)$ be the embedding map. The Sobolev embedding theorem implies that $i$ is linear compact. We have

$$
\left(K_{*}(i(v)), y\right)_{*}=\int_{\Omega} v y d z \quad \text { for all } y \in H^{1}(\Omega) \quad(\text { see }(2.8)) .
$$

The map $K_{*} \circ i$ is linear compact on $H^{1}(\Omega)$, self-adjoint and positive. So, from the well known spectral theorem for such operators (see, for example Gasinski 
and Papageorgiou [8, p. 296]), we can find $\left\{\mu_{n}\right\}_{n \geq 1}$ the sequence of distinct eigenvalues of $K_{*} \circ i$ such that

$$
\mu_{1}>\ldots>\mu_{n}>\ldots>0, \quad \mu_{n} \rightarrow 0^{+} \quad \text { as } n \rightarrow+\infty .
$$

Then $\widehat{\lambda}_{n}=1 / \mu_{n}-\widehat{\xi}$ for all $n \geq 1$ are the distinct eigenvalues of (2.2). So, we have

$$
-\infty<\hat{\lambda}_{1}<\ldots<\hat{\lambda}_{n}<\ldots, \quad \hat{\lambda}_{n} \rightarrow+\infty \quad \text { as } n \rightarrow+\infty .
$$

Also there is a corresponding sequence $\left\{\widehat{u}_{n}\right\}_{n \geq 1} \subseteq H^{1}(\Omega)$ of eigenfunctions such that $\left\{\widehat{u}_{n}\right\}_{n \geq 1}$ is an orthonormal basis of $L^{2}(\Omega)$ and an orthogonal basis of $H^{1}(\Omega)$. If $\beta \in L^{s}(\Omega), s>N$, then using the regularity results of Wang [21], we have $\left\{\widehat{u}_{n}\right\}_{n \geq 1} \subseteq C^{1}(\bar{\Omega})$. The eigenvalues have the following variational characterization (see $[8]$ ):

$$
\widehat{\lambda}_{1}=\inf \left[\frac{\sigma(u)}{\|u\|_{2}^{2}}: u \in H^{1}(\Omega), u \neq 0\right]
$$

(see Lemma 2.2),

$$
\begin{aligned}
\widehat{\lambda}_{k} & =\inf \left[\frac{\sigma(u)}{\|u\|_{2}^{2}}: u \in \overline{\bigoplus_{i \geq k} E\left(\widehat{\lambda}_{i}\right)}, u \neq 0\right] \\
& =\sup \left[\frac{\sigma(u)}{\|u\|_{2}^{2}}: u \in \bigoplus_{i=1}^{k} E\left(\widehat{\lambda}_{i}\right), u \neq 0\right]
\end{aligned}
$$

for $k \geq 2$. Here $E\left(\widehat{\lambda}_{i}\right)$ denotes the eigenspace corresponding to the eigenvalue $\widehat{\lambda}_{i}$. The infimum in (2.9) is realized on $E\left(\widehat{\lambda}_{1}\right)$ and both the infimum and the supremum in (2.10) are realized on $E\left(\widehat{\lambda}_{k}\right)$. The eigenvalue $\widehat{\lambda}_{1}$ is simple (i.e. $\left.\operatorname{dim} E\left(\widehat{\lambda}_{1}\right)=1\right)$ and it is clear from (2.9) that the eigenfunctions corresponding to $\widehat{\lambda}_{1}$ do not change sign. In fact $\widehat{\lambda}_{1}$ is the only eigenvalue with eigenfunctions of constant sign. All the other eigenvalues have nodal (sign-changing) eigenfunctions. In what follows, by $\widehat{u}_{1}$ we denote the positive $L^{2}$-normalized (i.e. $\|u\|_{2}=1$ ) eigenfunction corresponding to $\widehat{\lambda}_{1}$. If $\beta \in L^{s}(\Omega), s>N$, then $\widehat{u}_{1} \in C_{+} \backslash\{0\}$ (see Wang [21]) and by the Harnack inequality of Pucci and Serrin [16, p. 163] we have $\widehat{u}_{1}>0$ for all $z \in \Omega$. In fact if $\beta^{+} \in L^{\infty}(\Omega)$, then the boundary point theorem (see Pucci and Serrin [16, p. 120]), implies that $\widehat{u}_{1} \in$ int $_{+}$. When $\beta \in L^{s}(\Omega), s>N / 2$, the eigenspace $E\left(\widehat{\lambda}_{k}\right), k \geq 1$, have the so-called "Unique Continuation Property" (UCP for short) which says that, if $u \in E\left(\widehat{\lambda}_{k}\right)$ and $u$ vanishes on a set of positive Lebesgue measure, then $u \equiv 0$.

We can have a similar spectral analysis for a weighted version of the eigenvalue problem:

$$
-\triangle u(z)+\beta(z) u(z)=\lambda m(z) u(z), \quad \text { in } \Omega, \quad \frac{\partial u}{\partial n} \mid \partial \Omega=0 .
$$


as above, we can show that problem (2.11) admits a sequence $\left\{\widetilde{\lambda}_{k}(m)\right\}_{k \geq 1}$ of distinct eigenvalues such that $\widetilde{\lambda}_{1}(m)<\ldots<\widetilde{\lambda}_{k}(m)<\ldots$ and $\widetilde{\lambda}_{k}(m) \rightarrow+\infty$ as $k \rightarrow \infty$. These eigenvalues admit similar variational characterization using this time the Rayleigh quotient $\sigma(u) / \int_{\Omega} m u^{2} d z$ for all $u \in H^{1}(\Omega)$ (see (2.9) and (2.10)). We have the same properties for the corresponding eigenfunctions and eigenspaces. As an easy consequence of the UCP of the eigenspaces, we have the following monotonicity properties of the eigenvalues $\widetilde{\lambda}_{k}(m), k \geq 1$.

Proposition 2.3. If $m_{1}, m_{2} \in L^{\infty}(\Omega)_{+} \backslash\{0\}, 0 \leq m_{1}(z) \leq m_{2}(z)$ almost everywhere in $\Omega, m_{1} \neq m_{2}$, then $\widetilde{\lambda}_{k}\left(m_{2}\right)<\widetilde{\lambda}_{k}\left(m_{1}\right)$.

The following simple lemma will help us verify the mountain pass geometry for our problem.

LEMma 2.4. If $\theta \in L^{N / 2}(\Omega), \theta(z) \leq \widehat{\lambda}_{1}$ almost everywhere in $\Omega, \theta \neq \widehat{\lambda}_{1}$, then there exists $c^{*}>0$ such that

$$
\xi(u)=\sigma(u)-\int_{\Omega} \theta u^{2} d z \geq c^{*}\|u\|^{2} \quad \text { for all } u \in H^{1}(\Omega) .
$$

Proof. From (2.9) and Lemma 2.2 we have $\xi \geq 0$. We argue indirectly. So, suppose that the Lemma is not true. Exploiting the 2-homogeneity of $\xi(\cdot)$, we can find a sequence $\left\{u_{n}\right\}_{n \geq 1} \subseteq H^{1}(\Omega)$ such that

$$
\left\|u_{n}\right\|=1 \quad \text { for all } n \geq 1 \text { and } \xi\left(u_{n}\right) \rightarrow 0 \quad \text { as } n \rightarrow \infty .
$$

We may assume that

$$
u_{n} \rightarrow u \quad \text { in } H^{1}(\Omega) \quad \text { and } \quad u_{n} \rightarrow u \quad \text { in } L^{2^{*}}(\Omega) .
$$

The sequential weak lower semicontinuity of $\xi(\cdot)$ and (2.12), (2.13) imply

$$
\sigma(u) \leq \int_{\Omega} \theta u^{2} d z \leq \widehat{\lambda}_{1}\|u\|_{2}^{2}
$$

so from (2.9) we deduce $\sigma(u)=\widehat{\lambda}_{1}\|u\|_{2}^{2}$, hence

$$
u=\mu \widehat{u}_{1} \quad \text { with } \mu \in \mathbb{R} .
$$

If $\mu=0$, then $u_{n} \rightarrow 0$ in $H^{1}(\Omega)$, which contradicts the fact that $\left\|u_{n}\right\|=1$ for all $n \geq 1(\operatorname{see}(2.12))$. If $\mu \neq 0$, then $|u(z)|>0$ for almost all $z \in \Omega$ (by the UCP). So, from (2.14) and the hypothesis on $\theta$, we have $\sigma(u)<\widehat{\lambda}_{1}\|u\|_{2}^{2}$, which contradicts (2.9).

In a similar fashion exploiting the UCP, we also have the following result:

LEMMA 2.5 .

(a) If $\eta \in L^{\infty}(\Omega), \eta(z) \leq \widehat{\lambda}_{k}$ almost everywhere in $\Omega(k \geq 1)$ and $\eta \neq \widehat{\lambda}_{k}$, then there exists $\widetilde{c}>0$ such that

$$
\sigma(u)-\int_{\Omega} \eta u^{2} d z \geq \widetilde{c}\|u\|^{2} \quad \text { for all } u \in \overline{\bigoplus_{i \geq k} E\left(\widehat{\lambda}_{i}\right)} .
$$


(b) If $\eta \in L^{\infty}(\Omega), \eta(z) \geq \widehat{\lambda}_{k}$ almost everywhere in $\Omega(k \geq 1)$ and $\eta \neq \widehat{\lambda}_{k}$, then there exists $\widetilde{c}_{0}>0$ such that

$$
\sigma(u)-\int_{\Omega} \eta u^{2} d z \leq-\widetilde{c}_{0}\|u\|^{2} \quad \text { for all } u \in \bigoplus_{i=1}^{k} E\left(\widehat{\lambda}_{i}\right) .
$$

\section{Constant sign solutions}

In this section using variational methods based on the critical point theory, together with truncation and perturbation techniques, we produce two nontrivial constant sign solutions for problem (1.1).

In what follows by $m_{0} \geq 1$ we denote the first integer such that $\widehat{\lambda}_{m_{0}} \geq 0$ (i.e. is the first nonnegative eigenvalue). Evidently, if $\beta \geq 0$, then $m_{0}=1$.

The hypotheses on the data of (1.1) are the following:

$H(\beta) \quad \beta \in L^{s}(\Omega)$ with $s>N$ and $\beta^{+} \in L^{\infty}(\Omega)$.

$H(f)_{1} f: \Omega \times \mathbb{R} \rightarrow \mathbb{R}$ is a Carathéodory function such that $f(z, 0)=0$ for almost all $z \in \Omega$ and

(i) $|f(z, x)| \leq \alpha(z)(1+|x|)$ for almost all $z \in \Omega$, all $x \in \mathbb{R}$, with $\alpha \in L^{\infty}(\Omega)_{+}$

(ii) there exists an integer $m \geq \max \left\{m_{0}, 2\right\}$ such that

$$
\widehat{\lambda}_{m} \leq \liminf _{x \rightarrow \pm \infty} \frac{f(z, x)}{x} \leq \limsup _{x \rightarrow \pm \infty} \frac{f(z, x)}{x} \leq \widehat{\lambda}_{m+1}
$$

uniformly for almost all $z \in \Omega$;

(iii) if $F(z, x)=\int_{0}^{x} f(z, s) d s$, then there exist $\tau \in(0,2)$ and $\zeta \in L^{\infty}(\Omega)$, $\zeta(s) \leq 0$ almost everywhere in $\Omega, \zeta \neq 0$ such that

$$
\limsup _{x \rightarrow \pm \infty} \frac{f(z, x) x-2 F(z, x)}{|x|^{\tau}} \leq \zeta(z)
$$

uniformly for almost all $z \in \Omega$;

(iv) there exists a function $\theta \in L^{\infty}(\Omega)$ such that $\theta \leq \widehat{\lambda}_{1}$ for almost all $z \in \Omega, \theta \neq \widehat{\lambda}_{1}$ and

$$
\limsup _{x \rightarrow 0} \frac{f(z, x)}{x} \leq \theta(z)
$$

uniformly for almost all $z \in \Omega$;

(v) for every $\rho>0$ we can find $\xi_{\rho}>0$ such that $f(z, x) x+\xi_{\rho} x^{2} \geq 0$ for almost all $z \in \Omega$, all $|x| \leq \rho$.

REMARK 3.1. Hypothesis $H(f)_{1}$ (ii) implies that asymptotically at $\pm \infty$, the quotient $f(z, x) / x$ is in the spectral interval $\left[\widehat{\lambda}_{m}, \widehat{\lambda}_{m+1}\right]$ with possible interaction with both endpoints (double resonance). Hypothesis $H(f)_{1}$ (iv) implies that at the origin we have non uniform nonresonance with respect to the principal eigenvalue $\widehat{\lambda}_{1}>0$. 
We also consider the following perturbations-truncations of $f(z, \cdot)$ :

$$
\widehat{f}_{+}(z, x)= \begin{cases}0 & \text { if } x \leq 0 \\ f(z, x)+\widehat{\xi} x & \text { if } x>0\end{cases}
$$

and

$$
\widehat{f}_{-}(z, x)= \begin{cases}f(z, x)+\widehat{\xi} x & \text { if } x<0 \\ 0 & \text { if } x \geq 0\end{cases}
$$

Here $\widehat{\xi}>0$ is as in (2.7). Both $\widehat{f}_{ \pm}(z, x)$ are Carathéodory functions and we set $\widehat{F}_{ \pm}(z, x)=\int_{0}^{x} \widehat{f}_{ \pm}(z, s) d s$. We consider the $C^{1}$-functionals $\widehat{\varphi}_{ \pm}: H^{1}(\Omega) \rightarrow \mathbb{R}$ defined by

$$
\widehat{\varphi}_{ \pm}(u)=\frac{1}{2} \sigma(u)+\frac{\widehat{\xi}}{2}\|u\|_{2}^{2}-\int_{\Omega} \widehat{F}_{ \pm}(z, u(z)) d z \quad \text { for all } u \in H^{1}(\Omega) .
$$

Also, let $\varphi: H^{1}(\Omega) \rightarrow \mathbb{R}$ be the $C^{1}$-energy functional for problem (1.1) defined by

$$
\varphi(u)=\frac{1}{2} \sigma(u)-\int_{\Omega} F(z, u(z)) d z \quad \text { for all } u \in H^{1}(\Omega) .
$$

Proposition 3.2. If hypotheses $H(\beta)$ and $H(f)_{1}$ hold, then the functionals $\widehat{\varphi}_{ \pm}$satisfy the $C$-condition.

Proof. We do the proof for the functional $\widehat{\varphi}_{+}$, the proof for $\widehat{\varphi}_{-}$being similar. Let $\left\{u_{n}\right\}_{n \geq 1} \subseteq H^{1}(\Omega)$ be a sequence such that

$$
\left|\widehat{\varphi}_{+}\left(u_{n}\right)\right| \leq M_{1} \text { for some } M_{1}>0 \text {, all } n \geq 1
$$

and

$$
\left(1+\left\|u_{n}\right\|\right) \widehat{\varphi}_{+}^{\prime}\left(u_{n}\right) \rightarrow 0 \quad \text { in } H^{1}(\Omega)^{*}, \text { as } n \rightarrow \infty .
$$

From (3.4) we have

$$
\left|\left\langle A\left(u_{n}\right), h\right\rangle+\int_{\Omega}(\beta(z)+\widehat{\xi}) u_{n} h d z-\int_{\Omega} \widehat{f}_{+}\left(z, u_{n}\right) h d z\right| \leq \frac{\varepsilon_{n}\|h\|}{1+\left\|u_{n}\right\|}
$$

for all $h \in H^{1}(\Omega)$, with $\varepsilon_{n} \rightarrow 0^{+}$, where $A \in \mathcal{L}\left(H^{1}(\Omega), H^{1}(\Omega)^{*}\right)$ is defined by

$$
\langle A(u), v\rangle=\int_{\Omega}(D u, D v)_{\mathbb{R}^{N}} d z \quad \text { for all } u, v \in H^{1}(\Omega) .
$$

In (3.5) we choose $h=-u_{n}^{-} \in H^{1}(\Omega)$. Then $\sigma\left(u_{n}^{-}\right)+\widehat{\xi}\left\|u_{n}^{-}\right\|_{2}^{2} \leq \varepsilon_{n}$ for all $n \geq 1$ (see (3.1)-(3.2)). If we choose $\theta=-\widehat{\xi}$ in Lemma 2.4, we obtain $c^{*}\left\|u_{n}^{-}\right\|^{2} \leq \varepsilon_{n}$ for all $n \geq 1$, hence

$$
u_{n}^{-} \rightarrow 0 \quad \text { in } H^{1}(\Omega) .
$$

Then from (3.5) and (3.6), we have

$$
\left|\left\langle A\left(u_{n}^{+}\right), h\right\rangle+\int_{\Omega} \beta(z) u_{n}^{+} h d z-\int_{\Omega} f\left(z, u_{n}^{+}\right) h d z\right| \leq \varepsilon_{n}^{\prime}\|h\|
$$


with $\varepsilon_{n}^{\prime} \rightarrow 0^{+}$as $n \rightarrow \infty$. Suppose that $\left\|u_{n}^{+}\right\| \rightarrow+\infty$ and let $y_{n}=u_{n}^{+} /\left\|u_{n}^{+}\right\|$, $n \geq 1$. Then $\left\|y_{n}\right\|=1$ for all $n \geq 1$ and so we may assume that

$$
y_{n} \rightarrow y \text { in } H^{1}(\Omega) \text { and } y_{n} \rightarrow y \text { in } L^{s^{\prime}}(\Omega) \quad\left(\frac{1}{s}+\frac{1}{s^{\prime}}=1\right) \text { as } n \rightarrow \infty .
$$

From (3.7) we have

$$
\left|\left\langle A\left(y_{n}\right), h\right\rangle+\int_{\Omega} \beta(z) y_{n} h d z-\int_{\Omega} \frac{f\left(z, u_{n}^{+}\right)}{\left\|u_{n}^{+}\right\|} h d z\right| \leq \frac{\varepsilon_{n}^{\prime}\|h\|}{\left\|u_{n}\right\|} \quad \text { for all } n \geq 1 .
$$

By virtue of hypothesis $H(f)_{1}(\mathrm{i})$, we see that $N_{f}\left(u_{n}^{+}\right) /\left\|u_{n}^{+}\right\| \subseteq L^{2}(\Omega)$ is bounded. So, by passing to a subsequence if necessary and using hypothesis $H(f)_{1}(\mathrm{ii})$, we have

$$
\frac{N_{f}\left(u_{n}^{+}\right)}{\left\|u_{n}^{+}\right\|} \rightarrow \xi y \quad \text { in } L^{2}(\Omega) \text { with } \widehat{\lambda}_{m} \leq \xi(z) \leq \widehat{\lambda}_{m+1} \text { a.e. in } \Omega \text {. }
$$

Also, if in (3.9) we choose $h=y_{n}-y \in H^{1}(\Omega)$, pass to the limit as $n \rightarrow \infty$, use (3.8) and (3.10), we obtain

$$
\lim _{n \rightarrow \infty}\left\langle A\left(y_{n}\right), y_{n}-y\right\rangle=0, \quad \text { so } \quad\left\|D y_{n}\right\|_{2} \rightarrow\|D y\|_{2} .
$$

By the Kadec-Klee property of Hilbert spaces we have $y_{n} \rightarrow y$ in $H^{1}(\Omega)$, so

$$
\|y\|=1 \text {. }
$$

Now we pass to the limit as $n \rightarrow \infty$ in (3.9) and use (3.10), then

$$
\langle A(y), h\rangle+\int_{\Omega} \beta(z) y h d z=\int_{\Omega} \xi(z) y h d y, \quad \text { for all } h \in H^{1}(\Omega),
$$

that is $A(y)+\beta y=\xi y$, so

$$
-\triangle y(z)+\beta(z) y(z)=\xi(z) y(z) \quad \text { a.e. in } \Omega, \quad \frac{\partial y}{\partial n}=0 \quad \text { on } \partial \Omega .
$$

If $\xi \neq \widehat{\lambda}_{m}$ and $\xi(z) \neq \lambda_{m+1}$, then using Proposition 2.3 we have

$$
\tilde{\lambda}_{m}(\xi)<\widetilde{\lambda}_{m}\left(\widehat{\lambda}_{m}\right)=1 \text { and } 1=\widetilde{\lambda}_{m+1}\left(\widehat{\lambda}_{m_{+}+1}\right)<\widetilde{\lambda}_{m+1}(\xi) .
$$

From (3.12) and (3.13) we infer that $y=0$, which contradicts (3.11).

Now assume that $\xi=\widehat{\lambda}_{m}$ or $\xi=\widehat{\lambda}_{m+1}$. Then $y \in E\left(\widehat{\lambda}_{m}\right) \backslash\{0\}$ or $y \in$ $E\left(\widehat{\lambda}_{m+1}\right) \backslash\{0\}$ (see (3.11) and (3.12)). The UCP implies that $y(z)>0$ for almost all $z \in \Omega$ and so $u_{n}^{+}(z) \rightarrow+\infty$ for almsot all $z \in \Omega$. By virtue of hypothesis $H(f)_{1}$ (iii) and Fatou's lemma, we have

$$
\limsup _{n \rightarrow \infty} \frac{1}{\left\|u_{n}^{+}\right\|^{\tau}} \int_{\Omega}\left[f\left(z, u_{n}^{+}\right) u_{n}^{+}-2 F\left(z, u_{n}^{+}\right)\right] d z \leq \int_{\Omega} \xi(s) y(s) d s<0 .
$$

From (3.5) with $h=u_{n}^{+} \in H^{1}(\Omega)$, we have

$$
-\sigma\left(u_{n}^{+}\right)+\int_{\Omega} f\left(z, u_{n}^{+}\right) u_{n}^{+} d z \geq-\varepsilon_{n} \quad \text { for all } n \geq 1 .
$$


Also from (3.3) and (3.6) we have

$$
\sigma\left(u_{n}^{+}\right)-\int_{\Omega} 2 F\left(z, u_{n}^{+}\right) d z \geq-M_{1} \text { for all } n \geq 1 .
$$

Adding (3.15) and (3.16) and dividing by $\left\|u_{n}^{+}\right\|^{\tau}$, we obtain

$$
\frac{1}{\left\|u_{n}^{+}\right\|^{\tau}} \int_{\Omega}\left[f\left(z, u_{n}^{+}\right) u_{n}^{+}-2 F\left(z, u_{n}^{+}\right)\right] d z \geq-\frac{M_{2}}{\left\|u_{n}^{+}\right\|^{\tau}}
$$

for some $M_{2}>0$, all $n \geq 1$. Comparing (3.14) and (3.17) we reach a contradiction. This proves that $\left\{u_{n}^{+}\right\}_{n \geq 1} \subseteq H^{1}(\Omega)$ is bounded (see (3.6)). We may assume that

$$
u_{n} \rightarrow u \quad \text { in } H^{1}(\Omega) \quad \text { and } \quad u_{n} \rightarrow u \quad \text { in } L^{s^{\prime}}(\Omega) .
$$

In (3.5) we choose $h=u_{n}-u \in H^{1}(\Omega)$. Passing to the limit as $n \rightarrow \infty$ and using (3.18), we obtain

$$
\lim _{n \rightarrow \infty}\left\langle A\left(u_{n}\right), u_{n}-u\right\rangle=0
$$

so by the Kadec-Klee property of Hilbert spaces $u_{n} \rightarrow u$ in $H^{1}(\Omega)$. This proves that $\widehat{\varphi}_{+}$satisfies the $C$-condition. Similarly for the functional $\widehat{\varphi}_{-}$.

Minor changes in that above proof, lead to the following similar result for the functional $\varphi$.

Proposition 3.3. If hypotheses $H(\beta)$ and $H(f)_{1}$ hold, then the functional $\varphi$ satisfies the $C$-condition.

The next two propositions will verify the mountain pass geometry for the functionals $\widehat{\varphi}_{ \pm}$(see Theorem 2.1).

Proposition 3.4. If hypotheses $H(\beta)$ and $H(f)_{1}$ hold, then $\widehat{\varphi}_{ \pm}\left(t \widehat{u}_{1}\right) \rightarrow-\infty$ as $t \rightarrow \pm \infty$.

ProOF. By virtue of hypothesis $H(f)_{1}\left(\right.$ ii) given $\varepsilon \in\left(0, \widehat{\lambda}_{m}-\widehat{\lambda}_{1}\right)$, we can find $c_{1}=c_{1}(\varepsilon)>0$ such that

$$
F(z, x) \geq \frac{1}{2}\left(\widehat{\lambda}_{m}-\varepsilon\right) x^{2}+c_{1} \quad \text { for a.a. } z \in \Omega, \text { all } x \in \mathbb{R} .
$$

Then, for $t>0$, we have

$$
\widehat{\varphi}_{+}\left(t \widehat{u}_{1}\right)=\frac{t^{2}}{2} \sigma\left(\widehat{u}_{1}\right)+\frac{\widehat{\xi} t^{2}}{2}\left\|\widehat{u}_{1}\right\|_{2}^{2}-\int_{\Omega} \widehat{F}_{+}\left(z, t \widehat{u}_{1}\right) d z \leq \frac{t^{2}}{2}\left[\widehat{\lambda}_{1}-\widehat{\lambda}_{m}+\varepsilon\right]-c_{1}|\Omega|_{N}
$$

(see (3.19) and recall that $\left.\widehat{u}_{1} \in \operatorname{int} C_{+},\left\|\widehat{u}_{1}\right\|_{2}=1\right)$. Since $\varepsilon \in\left(0, \widehat{\lambda}_{m}-\widehat{\lambda}_{1}\right)$, we infer that $\widehat{\varphi}_{+}\left(t \widehat{u}_{1}\right) \rightarrow-\infty$ as $t \rightarrow+\infty$. Similarly for the functional $\widehat{\varphi}_{-}$.

Proposition 3.5. If hypotheses $H(\beta)$ and $H(f)_{1}$ hold, then $u=0$ is a local minimizer for the functionals $\widehat{\varphi}_{ \pm}$and $\varphi$. 
Proof. By virtue of hypothesis $H(f)_{1}(\mathrm{i})$, (iv), given $\varepsilon>0$ and $r>2$, we can find $c_{2}=c_{2}(\varepsilon, r)>0$ such that

$$
F(z, x) \leq \frac{1}{2}(\theta(z)+\varepsilon) x^{2}+c_{2}|x|^{r} \quad \text { for a.a. } z \in \Omega, \text { all } x \in \mathbb{R} .
$$

Then, for every $u \in H^{1}(\Omega)$, we have

$$
\begin{aligned}
\widehat{\varphi}_{+}(u) & =\frac{1}{2} \sigma(u)+\frac{\widehat{\xi}}{2}\|u\|_{2}^{2}-\int_{\Omega} \widehat{F}_{+}(z, u) d z \\
& \geq \frac{1}{2}\left[\sigma(u)-\int_{\Omega} \theta u^{2} d z\right]-\frac{\varepsilon}{2}\|u\|_{2}^{2}-c_{3}\|u\|^{r} \geq \frac{c_{*}-\varepsilon}{2}\|u\|^{2}-c_{3}\|u\|^{r},
\end{aligned}
$$

for some $c_{3}, c_{*}>0$ (see $(3.20)$ and $\left.(3.1)\right)$. Choosing $\varepsilon \in\left(0, c_{*}\right)$, we have

$$
\widehat{\varphi}_{+}(u) \geq c_{4}\|u\|^{2}-c_{3}\|u\|^{r} \quad \text { with } c_{4}=c_{*}-\varepsilon>0 .
$$

Since $r>2$, we ca find $\rho \in(0,1)$ small such that $\widehat{\varphi}_{+}(u)>0$ for all $u \in H^{1}(\Omega)$, with $0<\|u\| \leq \rho$, so $u=0$ is a strict local minimizer of $\widehat{\varphi}_{+}$. Similarly for the functionals $\widehat{\varphi}$ - and $\varphi$.

Now we are ready to produce nontrivial constant sign solutions.

Proposition 3.6. If hypotheses $H(\beta)$ and $H(f)_{1}$ hold, then problem (1.1) has at least two nontrivial constant sign solutions

$$
u_{0} \in \operatorname{int} C_{+} \quad \text { and } \quad v_{0} \in-\operatorname{int} C_{+} .
$$

Proof. From Proposition 3.5 and its proof, we know that we can find $\rho \in$ $(0,1)$ small such that

$$
\widehat{\varphi}_{+}(0)=0<\inf \left[\widehat{\varphi}_{+}(u):\|u\|=\rho\right]=\widehat{\eta}_{\rho}^{+} .
$$

Then (3.22) combined with Propositions 3.2 and 3.4, permit the use of Theorem 2.1. so, we can find $u_{0} \in H^{1}(\Omega)$ such that

$$
\widehat{\varphi}_{+}^{\prime}\left(u_{0}\right)=0 \text { and } \widehat{\eta}_{\rho}^{+} \leq \widehat{\varphi}_{+}\left(u_{0}\right) .
$$

From (3.22) and (3.23), we see that $u_{0} \neq 0$. From (3.23) we also have

$$
A\left(u_{0}\right)+(\beta+\widehat{\xi}) u_{0}=N_{\widehat{f}_{+}}\left(u_{0}\right) .
$$

On (3.24) we act with $-u_{0}^{-} \in H^{1}(\Omega)$. Then (3.1) forces $\sigma\left(u_{0}^{-}\right)+\widehat{\xi}\left\|u_{0}^{-}\right\|_{2}^{2}=0$, so by (2.7) we obtain $\left\|u_{0}^{-}\right\|^{2} \leq 0$, hence $u_{0} \geq 0, u_{0} \neq 0$. Therefore (3.24) becomes $A\left(u_{0}\right)+\beta u_{0}=N_{f}\left(u_{0}\right)$, so

(3.25) $-\triangle u_{0}(z)+\beta(z) u_{0}(z)=f\left(z, u_{0}(z)\right) \quad$ a.e. in $\Omega, \quad \frac{\partial u_{0}}{\partial n}=0 \quad$ on $\partial \Omega$.

Hence $u_{0} \in H^{1}(\Omega)$ is a nontrivial positive solution of (1.1). Hypotheses $H(f)_{1}(\mathrm{i})$, (iv), (v) imply that

$$
|f(z, x)| \leq c_{5}|x| \quad \text { for a.a. } z \in \Omega \text {, all } x \in \mathbb{R} \text { and some } c_{5}>0 .
$$


We set

$$
h(z)= \begin{cases}\frac{f\left(z, u_{0}(z)\right)}{u_{0}(z)} & \text { if } u_{0}(z) \neq 0 \\ 0 & \text { if } u_{0}(z)=0 .\end{cases}
$$

From (3.26) we see that $h \in L^{\infty}(\Omega)$. from (3.25) we have

$$
-\triangle u_{0}(z)=(h(z)-\beta(z)) u_{0}(z) \quad \text { a.e. in } \Omega, \quad \frac{\partial u_{0}}{\partial n}=0 \quad \text { on } \partial \Omega .
$$

Note that $(h-\beta)(\cdot) \in L^{s}(\Omega)$. Invoking Lemma 5.1 of [21] we have that $u_{0} \in$ $L^{\infty}(\Omega)$. From (3.27) it follows that $-\triangle u_{0} \in L^{s}(\Omega)$. Then by virtue of Lemma 5.2 of [21] we have $u_{0} \in H^{2, s}(\Omega)$. Since $s>N$, the Sobolev embedding theorem implies that $H^{2, s}(\Omega) \hookrightarrow C^{1, \alpha}(\bar{\Omega})$ with $\alpha=1-N / s>0$. Therefore $u_{0} \in C_{+} \backslash\{0\}$. From (3.27) we have

$$
\triangle u_{0}(z)=(\beta(z)-h(z)) u_{0}(z) \leq\left(\beta^{+}(z)-h(z)\right) u_{0}(z) \text { a.e. in } \Omega,
$$

hence

$$
\triangle u_{0}(z) \leq\left(\left\|\beta^{+}\right\|_{\infty}+\|h\|_{\infty}\right) u_{0}(z) \text { a.e. in } \Omega,
$$

so, from Vazquez [20] we obtain $u_{0} \in \operatorname{int} C_{+}$.

Similarly, working this time with the functional $\widehat{\varphi}$ - we produce a second nontrivial constant sign solution $v_{0} \in-\operatorname{int} C_{+}$.

\section{Multiplicity theorems}

In this section we prove the two multiplicity theorems for problem (1.1). As we already mentioned in the Introduction, our approach will be based on the reduction method (see Amann [1], Castro-Lazer [4] and Thews [19]).

Let $Y=\bigoplus_{i=1}^{m} E\left(\widehat{\lambda}_{i}\right), \widehat{H}=Y^{\perp}=\overline{\bigoplus_{i \geq m+1} E\left(\widehat{\lambda}_{i}\right)}$. We have the following orthogonal direct sum decomposition:

$$
H^{1}(\Omega)=Y \oplus \widehat{H}
$$

So, every $u \in H^{1}(\Omega)$ admits a unique sum decomposition

$$
u=y+\widehat{u} \quad \text { with } y \in Y, \widehat{u} \in \widehat{H} .
$$

To implement the reduction method, we need to strengthen the conditions on the reaction $f(z, x)$ :

$H(f)_{2} \quad f: \Omega \times \mathbb{R} \rightarrow \mathbb{R}$ is a measurable function such that $f(z, 0)=0$ for almost all $z \in \Omega$ and

(i) there exists integer $m \geq \max \left\{m_{0}, 2\right\}$ and a function $\eta \in L^{\infty}(\Omega)$ such that

$$
\eta(z) \leq \widehat{\lambda}_{m+1} \quad \text { a.a. in } \Omega, \eta \neq \widehat{\lambda}_{m+1},
$$

and $|f(z, x)-f(z, y)| \leq \eta(z)|x-y|$ for almost all $z \in \Omega$, all $x, y \in \mathbb{R}$; 
(ii) $\widehat{\lambda}_{m} \leq \liminf _{x \rightarrow \pm \infty} f(z, x) / x$ uniformly for almost all $z \in \Omega$;

(iii) there exist $\mu \in(0,2)$ and $\zeta \in L^{\infty}(\Omega), \zeta(z) \leq 0$ almost everywhere in $\Omega, \zeta \neq 0$ such that

$$
\limsup _{x \rightarrow \pm \infty} \frac{f(z, x) x-2 F(z, x)}{|x|^{\mu}} \leq \zeta(z) \text { uniformly for a.a. } z \in \Omega \text {; }
$$

(iv) there exists a function $\theta \in L^{\infty}(\Omega)$ such that

$$
\theta(z) \leq \widehat{\lambda}_{1} \quad \text { for a.a. } z \in \Omega, \theta \neq \widehat{\lambda}_{1}
$$

and

$$
\limsup _{x \rightarrow 0} \frac{f(z, x)}{x} \leq \theta(z) \quad \text { uniformly for a.a. } z \in \Omega
$$

(v) for every $\rho>0$ we can find $\xi_{\rho}>0$ such that

$$
f(z, x) x+\xi_{\rho} x^{2} \geq 0 \quad \text { for a.a. } z \in \Omega \text {, all }|x| \leq \rho .
$$

REMARK 4.1. By virtue of hypotheses $H(f)_{2}(\mathrm{i})$, (ii) we have

(4.1) $\widehat{\lambda}_{m} \leq \liminf _{x \rightarrow \pm \infty} \frac{f(z, x)}{x} \leq \limsup _{x \rightarrow \pm \infty} \frac{f(z, x)}{x} \leq \eta(z) \quad$ uniformly for a.a. $z \in \Omega$.

Proposition 4.2. If hypotheses $H(\beta)$ and $H(f)_{2}$ hold, then there exists a continuous map $\xi^{*}: Y \rightarrow \widehat{H}$ such that

$$
\inf [\varphi(y+\widehat{u}): \widehat{u} \in \widehat{H}]=\varphi\left(y+\xi^{*}(y)\right) \quad \text { for all } y \in Y .
$$

Proof. Fix $y \in Y$ and consider the $C^{1}$-functional $\varphi_{y}: H^{1}(\Omega) \rightarrow \mathbb{R}$ defined by

$$
\varphi_{y}(u)=\varphi(y+u) \quad \text { for all } u \in H^{1}(\Omega) .
$$

Let $i: \widehat{H} \rightarrow \mathbb{R}$ be the inclusion map and let $\widehat{\varphi}_{y}=\varphi_{y} \circ i: \widehat{H} \rightarrow \mathbb{R}$. By the chain rule we have $\widehat{\varphi}_{y}^{\prime}(\widehat{u})=p_{\widehat{H}^{*}} \varphi_{y}^{\prime}(\widehat{u})$ for all $\widehat{u} \in \widehat{H}$, where $p_{\widehat{H}^{*}}$ is the orthogonal projection of the Hilbert space $H^{1}(\Omega)^{*}$ onto $\widehat{H}^{*}$. If we take into account $H(f)_{2}(\mathrm{i})$ and Lemma 2.5, then for $\widehat{u}_{1}, \widehat{u}_{2} \in \widehat{H}$ we have

$$
\begin{aligned}
&\left\langle\widehat{\varphi}_{y}^{\prime}\left(\widehat{u}_{1}\right)-\widehat{\varphi}_{y}^{\prime}\left(\widehat{u}_{2}\right), \widehat{u}_{1}-\widehat{u}_{2}\right\rangle_{\widehat{H}}=\left\langle A\left(\widehat{u}_{1}-\widehat{u}_{2}\right), \widehat{u}_{1}-\widehat{u}_{2}\right\rangle \\
&+\int_{\Omega} \beta(z)\left(\widehat{u}_{1}-\widehat{u}_{2}\right)^{2} d z-\int_{\Omega}\left(f\left(z, y+\widehat{u}_{1}\right)-f\left(z, y+\widehat{u}_{2}\right)\right)\left(\widehat{u}_{1}-\widehat{u}_{2}\right) d z \\
& \quad \geq \sigma\left(\widehat{u}_{1}-\widehat{u}_{2}\right)-\int_{\Omega} \eta(z)\left(\widehat{u}_{1}-\widehat{u}_{2}\right)^{2} d z \geq c_{6}\left\|\widehat{u}_{1}-\widehat{u}_{2}\right\|^{2}
\end{aligned}
$$

for some $c_{6}>0$, so $\hat{\varphi}_{y}^{\prime}$ is strongly monotone, hence $\widehat{\varphi}_{y}$ is strictly convex. Also, we have

$$
\left\langle\widehat{\varphi}_{y}^{\prime}(\widehat{u}), \widehat{u}\right\rangle=\left\langle\hat{\varphi}_{y}^{\prime}(\widehat{u})-\widehat{\varphi}_{y}^{\prime}(0), \widehat{u}\right\rangle+\left\langle\widehat{\varphi}_{y}^{\prime}(0), \widehat{u}\right\rangle \geq c_{6}\|\widehat{u}\|^{2}-c_{7}\|\widehat{u}\|
$$

for some $c_{7}>0$, hence $\hat{\varphi}_{y}^{\prime}$ is coercive. Since $\hat{\varphi}_{y}^{\prime}$ is continuous and strongly monotone, it is maximal monotone. But a maximal monotone coercive map is 
surjective (see, for example Gasinski and Papageorgiou [8, p. 320]). So, we can find $\widehat{u}_{0} \in \widehat{H}$ such that

$$
\widehat{\varphi}_{y}^{\prime}\left(\widehat{u}_{0}\right)=0 .
$$

Then $\widehat{u}_{0}$ is unique (by virtue of the strong monotonicity of $\widehat{\varphi}_{y}^{\prime}$ ) and it is the unique global minimizer of the strictly convex functional $\widehat{\varphi}_{y}$. So, we can define the map $\xi^{*}: Y \rightarrow \widehat{H}$ which to each $y \in Y$ assign the unique global minimizer $\widehat{u}_{0} \in \widehat{H}$ of $\widehat{\varphi}_{y}$. We have

$$
\begin{aligned}
0=\widehat{\varphi}_{y}^{\prime}\left(\xi^{*}(y)\right) & =p_{\widehat{H}^{*}} \varphi^{\prime}\left(y+\xi^{*}(y)\right) \\
\varphi\left(y+\xi^{*}(y)\right) & =\inf [\varphi(y+\widehat{u}): \widehat{u} \in \widehat{H}] .
\end{aligned}
$$

Let $y_{n} \rightarrow y$ in $Y$. Then from (4.2)-(4.4), we have that $\left\{\xi^{*}\left(y_{n}\right)\right\}_{n \geq 1} \subseteq \widehat{H} \subseteq$ $H^{1}(\Omega)$ is bounded. So, we may assume that $\xi^{*}\left(y_{n}\right) \rightarrow v$ in $\subseteq H^{1}(\Omega)$. Using the Sobolev embedding theorem, we can easily check that $\varphi$ is sequentially weakly lower semicontinuous. So, we have

$$
\varphi(y+v) \leq \liminf _{n \rightarrow \infty} \varphi\left(y_{n}+\xi^{*}\left(y_{n}\right)\right) .
$$

But from (4.3) we have

$$
\varphi\left(y_{n}+\xi^{*}\left(y_{n}\right)\right) \leq \varphi\left(y_{n}+\widehat{u}\right) \quad \text { for all } \widehat{u} \in \widehat{H},
$$

so, bearing in mind (4.5) and the convergence of $\left\{y_{n}\right\}$ to $y$ we deduce

$$
\varphi(y+v) \leq \varphi(y+\widehat{u}) \text { for all } \widehat{u} \in \widehat{H},
$$

hence (4.3) yields $v=\xi^{*}(y)$. From (4.3) we have

$$
p_{\widehat{H}^{*}} \varphi^{\prime}\left(y_{n}+\xi^{*}\left(y_{n}\right)\right)=0 \text { for all } n \geq 1,
$$

that is,

$$
p_{\widehat{H}^{*}}\left[A\left(y_{n}+\xi^{*}\left(y_{n}\right)\right)+\beta\left(y_{n}+\xi^{*}\left(y_{n}\right)\right)\right]=p_{\widehat{H}^{*}} N_{f}\left(y_{n}+\xi^{*}\left(y_{n}\right)\right)
$$

for all $n \geq 1$, hence

$$
\lim _{n \rightarrow \infty}\left\langle A\left(y_{n}+\xi^{*}\left(y_{n}\right), \xi^{*}\left(y_{n}\right)-\xi^{*}(y)\right\rangle=0,\right.
$$

so by the Kadec-Klee property of Hilbert spaces $\xi^{*}\left(y_{n}\right) \rightarrow \xi^{*}(y)$ and $\xi^{*}$ is continuous.

Let $\widetilde{\varphi}: Y \rightarrow \mathbb{R}$ be the functional defined by

$$
\widetilde{\varphi}(y)=\varphi\left(y+\xi^{*}(y) \text { for all } y \in Y .\right.
$$

Proposition 4.3. If hypotheses $H(\beta)$ and $H(f)_{2}$ hold, then $\widetilde{\varphi} \in C^{1}(Y)$. 
Proof. Let $y, v \in Y$ and $\lambda>0$ (the analysis is similar if $\lambda<0$ ). Then

$$
\frac{\widetilde{\varphi}(y+\lambda v)-\widetilde{\varphi}(y)}{\lambda} \leq \frac{\varphi\left(y+\lambda v+\xi^{*}(y)\right)-\varphi\left(y+\xi^{*}(y)\right)}{\lambda},
$$

so

$$
\limsup _{\lambda \rightarrow 0} \frac{\tilde{\varphi}(y+\lambda v)-\widetilde{\varphi}(y)}{\lambda} \leq\left\langle\varphi^{\prime}\left(y+\xi^{*}(y)\right), v\right\rangle .
$$

Also, we have

$$
\frac{\widetilde{\varphi}(y+\lambda v)-\widetilde{\varphi}(y)}{\lambda} \geq \frac{\varphi\left(y+\lambda v+\xi^{*}(y+\lambda v)\right)-\widetilde{\varphi}\left(y+\xi^{*}(y+\lambda v)\right)}{\lambda},
$$

so

$$
\liminf _{\lambda \rightarrow 0} \frac{\widetilde{\varphi}(y+\lambda v)-\widetilde{\varphi}(y)}{\lambda} \geq\left\langle\varphi^{\prime}\left(y+\xi^{*}(y)\right), v\right\rangle .
$$

From (4.6) and (4.7), we see that $\widetilde{\varphi}$ is Gateaux differentiable at $y \in Y$ and

$$
\left\langle\widetilde{\varphi}_{G}^{\prime}(y), v\right\rangle_{Y}=\left\langle\varphi^{\prime}\left(y+\xi^{*}(y)\right), i_{Y}(v)\right\rangle \text { for all } v \in Y,
$$

where $i_{Y}: Y \rightarrow H^{1}(\Omega)$ is the inclusion map; so,

$$
\widetilde{\varphi}_{G}^{\prime}(y)=p_{Y^{*}} \varphi^{\prime}\left(y+\xi^{*}(y)\right) .
$$

From Proposition 4.2 we know that the map $y \rightarrow \widetilde{\varphi}_{G}^{\prime}(y)$ is continuous, so $\widetilde{\varphi} \in$ $C^{1}(Y)$.

REMARK 4.4. The above proposition allows us to consider the critical groups of $\widetilde{\varphi}$ at any isolated critical point.

Proposition 4.5. If hypotheses $H(\beta)$ and $H(f)_{2}$ hold, then $\widetilde{\varphi}$ is anticoercive, i.e. if $\|y\| \rightarrow \infty, y \in Y$, then $\widetilde{\varphi}(y) \rightarrow-\infty$.

Proof. We argue by contradiction. so, suppose we could find $\left\{y_{n}\right\}_{n \geq 1} \subseteq Y$ and $M_{3}>0$ such that

$$
\left\|y_{n}\right\| \rightarrow \infty \quad \text { and } \quad-M_{3} \leq \widetilde{\varphi}\left(y_{n}\right) \text { for all } n \geq 1 .
$$

We have

$$
-M_{3} \leq \widetilde{\varphi}\left(y_{n}\right) \leq \varphi\left(y_{n}\right)=\frac{1}{2} \sigma\left(y_{n}\right)-\int_{\Omega} F\left(z, y_{n}\right) d z .
$$

Let $h_{n}=y_{n} /\left\|y_{n}\right\|, n \geq 1$. Then $h_{n} \in Y$ and $\left\|h_{n}\right\|=1$ for all $n \geq 1$. The finite dimensionality of $Y$ implies that by passing to a subsequence if necessary, we have $h_{n} \rightarrow h$ in $Y$, and $\|h\|=1$. From (4.8) we have

$$
-\frac{M_{3}}{\left\|y_{n}\right\|^{2}} \leq \frac{1}{2} \sigma\left(h_{n}\right)-\int_{\Omega} \frac{F\left(z, y_{n}\right)}{\left\|y_{n}\right\|^{2}} d z \quad \text { for all } n \geq 1 .
$$

By virtue of (4.1) we have

$$
\widehat{\lambda}_{m} \leq \liminf _{x \rightarrow \pm \infty} \frac{2 F(z, x)}{x^{2}} \leq \limsup _{x \rightarrow \pm \infty} \frac{2 F(z, x)}{x^{2}} \leq \eta(z)
$$


uniformly for almost all $z \in \Omega$. Recall that from (3.26) we have $|F(z, x)| \leq c_{8} x^{2}$ for almost all $z \in \Omega$, all $x \in \mathbb{R}$ and some $c_{8}>0$, so

$$
\left\{\frac{F\left(\cdot, y_{n}(\cdot)\right)}{\left\|y_{n}\right\|^{2}}\right\}_{n \geq 1} \subseteq L^{1}(\Omega) \text { is uniformly integrable. }
$$

By virtue of the Dunford-Pettis theorem and because of (4.10), we have

$$
\frac{F\left(\cdot, y_{n}(\cdot)\right)}{\left\|y_{n}\right\|^{2}} \rightarrow \frac{1}{2} \eta_{0} h^{2} \quad \text { in } L^{1}(\Omega), \text { with } \widehat{\lambda}_{m} \leq \eta_{0} \leq \eta .
$$

So, if in (4.9) we pass to the limit as $n \rightarrow \infty$ and use (4.11), then

$$
0 \leq \frac{1}{2} \sigma(h)-\frac{1}{2} \int_{\Omega} \eta_{0} h^{2} d z \leq 0 .
$$

If $\eta_{0} \neq \widehat{\lambda}_{m}$, then, from Lemma 2.5 we obtain

$$
0 \leq \sigma(h)-\int_{\Omega} \eta_{0} h^{2} d z \leq-\widetilde{c}_{0}\|h\|^{2},
$$

a contradiction. So, we may assume that $\eta_{0} \equiv \widehat{\lambda}_{m}$. Then $h \in E\left(\widehat{\lambda}_{m}\right)$ and so by the UCP we have $h(z) \neq 0$ for almsot all $z \in \Omega$. Hence $\left|y_{n}(z)\right| \rightarrow+\infty$ for almost all $z \in \Omega$. By virtue of hypothesis $H(f)_{2}(\mathrm{iii})$, given any $\varepsilon>0$, we can find $M_{4}=M_{4}(\varepsilon)>0$ such that

$$
f(z, x) x-2 F(z, x) \leq(\zeta(s)+\varepsilon)|x|^{\mu} \quad \text { for a.a. } z \in \Omega, \text { all }|x| \geq M_{4} .
$$

Then we have

$$
\frac{d}{d x} \frac{F(z, x)}{x^{2}}=\frac{f(z, x) x-2 F(z, x)}{x^{3}} \leq \frac{\zeta(s)+\varepsilon}{x^{3-\mu}} \text { for a.a. } z \in \Omega, \text { all } x \geq M_{4},
$$

$\mathrm{SO}$

$\frac{F(z, x)}{x^{2}}-\frac{F(z, v)}{v^{2}} \leq-\frac{\zeta(s)+\varepsilon}{2-\mu}\left[\frac{1}{x^{2-\mu}}-\frac{1}{v^{2-\mu}}\right]$ for a.a. $z \in \Omega$, all $x \geq v \geq M_{4}$,

Let $x \rightarrow+\infty$. Then from (4.10) we have

$$
\frac{\widehat{\lambda}_{m}}{2} v^{2}-F(z, v) \leq \frac{\zeta(s)+\varepsilon}{2-\mu} v^{\mu} \quad \text { for a.a. } z \in \Omega \text {, all } v \geq M_{4},
$$

so

$$
\limsup _{v \rightarrow+\infty} \frac{1}{v^{\mu}}\left[\frac{\widehat{\lambda}_{m}}{2} v^{2}-F(z, v)\right] \leq \frac{\zeta(s)+\varepsilon}{2-\mu} \text { for a.a. } z \in \Omega .
$$

Similarly, we show that

$$
\limsup _{v \rightarrow-\infty} \frac{1}{|v|^{\mu}}\left[\frac{\widehat{\lambda}_{m}}{2} v^{2}-F(z, v)\right] \leq \frac{\zeta(s)+\varepsilon}{2-\mu} \quad \text { for a.a. } z \in \Omega .
$$

Therefore

$$
\limsup _{|v| \rightarrow \infty} \frac{1}{|v|^{\mu}}\left[\frac{\widehat{\lambda}_{m}}{2} v^{2}-F(z, v)\right] \leq \frac{\zeta(s)+\varepsilon}{2-\mu} \quad \text { for a.a. } z \in \Omega .
$$


From (4.8) and (2.10), we have

$$
-M_{3} \leq \frac{1}{2} \sigma\left(y_{n}\right)-\int_{\Omega} F\left(z, y_{n}\right) d z \leq \int_{\Omega}\left[\frac{\hat{\lambda}_{m}}{2} y_{n}^{2}-F\left(z, y_{n}\right)\right] d z .
$$

Since $\left|y_{n}(z)\right| \rightarrow+\infty$ for almost all $z \in \Omega$, from (4.13), Fatou's lemma, (4.14) and choosing $\varepsilon \in\left(0,-\int_{\Omega} \zeta(s) d s\right)$, we reach a contradiction. This proves the anticoercivity of $\widetilde{\varphi}$.

REMARK 4.6. In particular the above proposition implies that $\widetilde{\varphi}$ satisfies the C-condition (just note that $-\widetilde{\varphi}$ is coercive).

Now we are ready for the first multiplicity theorem concerning problem (1.1).

THEOREM 4.7. If hypotheses $H(\beta)$ and $H(f)_{2}$ hold, then problem (1.1) has at least three nontrivial solutions

$$
u_{0} \in \operatorname{int} C_{+}, \quad v_{0} \in-\operatorname{int} C_{+} \quad \text { and } \quad y_{0} \in C^{1}(\bar{\Omega}) .
$$

Proof. From Proposition 3.6, we already have two nontrivial constant sign solutions

$$
u_{0} \in \operatorname{int} C_{+} \quad \text { and } \quad v_{0} \in-\operatorname{int} C_{+} .
$$

From the proof of Proposition 3.6 we know that $u_{0}$ is a critical point of $\hat{\varphi}_{+}$of mountain pass type and $v_{0}$ is a critical point of $\widehat{\varphi}_{-}$of mountain pass type. Hence

$$
C_{1}\left(\widehat{\varphi}_{+}, u_{0}\right) \neq 0 \text { and } C_{1}\left(\widehat{\varphi}_{-}, v_{0}\right) \neq 0 .
$$

Note that from $(3.1)$ we have $\widehat{\varphi}_{+\mid C_{+}}=\varphi_{\mid C_{+}}$and $\widehat{\varphi}_{-\mid-C_{+}}=\varphi_{\mid-C_{+}}$. Since $u_{0} \in \operatorname{int} C_{+}$and $v_{0} \in-\operatorname{int} C_{+}$, we have

$$
\begin{aligned}
& C_{k}\left(\widehat{\varphi}_{+\mid C^{1}(\bar{\Omega})}, u_{0}\right)=C_{k}\left(\varphi_{\mid C^{1}(\bar{\Omega})}, u_{0}\right), \\
& C_{k}\left(\widehat{\varphi}_{-\mid C^{1}(\bar{\Omega})}, v_{0}\right)=C_{k}\left(\varphi_{\mid C^{1}(\bar{\Omega})}, v_{0}\right) .
\end{aligned}
$$

From Proposition 2.6 of Bartsch [3] and for $w \in\left\{u_{0}, v_{0}\right\}$, we have

$$
C_{k}\left(\widehat{\varphi}_{ \pm \mid C^{1}(\bar{\Omega})}, w\right)=C_{k}\left(\widehat{\varphi}_{ \pm}, w\right) \quad \text { and } \quad C_{k}\left(\varphi_{\mid C^{1}(\bar{\Omega})}, w\right)=C_{k}(\varphi, w)
$$

for all $k \geq 1$. Then from (4.15)-(4.18) it follows that

$$
C_{1}\left(\varphi, u_{0}\right) \neq 0 \quad \text { and } \quad C_{1}\left(\varphi, v_{0}\right) \neq 0 .
$$

Let $p_{Y}$ be orthogonal projection of $H^{1}(\Omega)$ onto $Y$. From Liu and Li [13] we have that $\bar{u}_{0}=p_{Y}\left(u_{0}\right) \in K_{\widetilde{\varphi}}, \bar{v}_{0}=p_{Y}\left(v_{0}\right) \in K_{\widetilde{\varphi}}$ and

$$
C_{k}\left(\varphi, u_{0}\right)=C_{k}\left(\widetilde{\varphi}, \bar{u}_{0}\right), \quad C_{k}\left(\varphi, v_{0}\right)=C_{k}\left(\widetilde{\varphi}, \bar{v}_{0}\right), \quad \text { for all } k \geq 0 .
$$

It follows from (4.19) that

$$
C_{1}\left(\widetilde{\varphi}, \bar{u}_{0}\right) \neq 0 \quad \text { and } \quad C_{1}\left(\widetilde{\varphi}, \bar{v}_{0}\right) \neq 0 .
$$


From Proposition 4.5 we know that $\widetilde{\varphi}$ is anticoercive on $Y$. Also, it is continuous. So, by virtue of the Weierstrass theorem we can find $\bar{y}_{0}$ a maximizer of $\widetilde{\varphi}$. Hence

$$
C_{k}\left(\widetilde{\varphi}, \bar{y}_{0}\right)=\delta_{k, d_{m}} Z \text { for all } k \geq 0 \text { where } d_{m}=\operatorname{dim} Y \geq 2 \text {. }
$$

Finally from Proposition 3.5 and from Liu and $\mathrm{Li}$ [13], we have

$$
C_{k}(\varphi, 0)=\delta_{k, 0} Z \text { for all } k \geq 0,
$$

so $C_{k}(\widetilde{\varphi}, 0)=\delta_{k, 0} Z$ for all $k \geq 0$. Comparing (4.20)-(4.22), we see that $\bar{y}_{0} \notin$ $\left\{0, \bar{u}_{0}, \bar{v}_{0}\right\}$. Then $y_{0}=\bar{y}_{0}+\xi^{*}\left(\bar{y}_{0}\right) \in H^{1}(\Omega)$ is a nontrivial critical point of $\varphi$ distinct from $u_{0}$ and $v_{0}$. Therefore $y_{0}$ is a third nontrivial solution of (1.1) and as before (see the proof of Proposition 3.6), using the regularity results of Wang [21], we have that $y_{0} \in C^{1}(\bar{\Omega})$.

Next, by strengthening the regularity of $f(z, \cdot)$, we will produce a fourth nontrivial solution. To this end, we need to compute the critical groups of $\widetilde{\varphi}$ at infinity. To do this, we do not need the stronger conditions on $f(z, x)$. So, using some ideas of Liu [12], we are able to compute precisely the critical groups of $\widetilde{\varphi}$ at infinity. In what follows, we assume that $K_{\widetilde{\varphi}}$ is finite or otherwise we already have infinitely many solutions of (1.1) and so we are done.

Proposition 4.8. If hypotheses $H(\beta)$ and $H(f)_{2}$ hold, then $C_{k}(\widetilde{\varphi}, \infty)=$ $\delta_{k, d_{m}} Z$ for all $k \geq 0$, where $d_{m}=\operatorname{dim} Y=\operatorname{dim} \bigoplus_{i=1}^{m} E\left(\widehat{\lambda}_{i}\right) \geq 2$.

Proof. Let $\mu_{0}<\inf \widetilde{\varphi}\left(K_{\widetilde{\varphi}}\right)$. From Proposition 4.5 we know that $\widetilde{\varphi}$ is anticoercive. So, we can find $\gamma<\xi<\mu_{0}$ and $0<\rho<R$ such that

$$
C_{R} \subseteq \widetilde{\varphi}^{\gamma} \subseteq C_{\rho} \subseteq \tilde{\varphi}^{\xi},
$$

where for every $r>0, C_{r}=\{y \in Y:\|y\| \geq r\}$.

We consider the long exact sequences of singular homology groups corresponding to triples $\left(C_{R}, C_{\rho}, Y\right)$ and $\left(\widetilde{\varphi}^{\gamma}, \widetilde{\varphi}^{\xi}, Y\right)$. So, we have

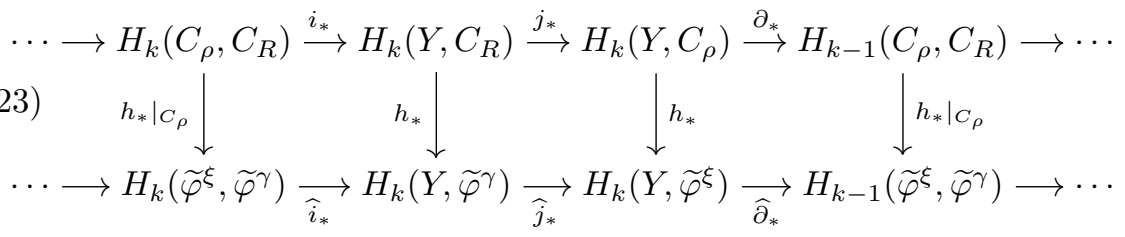

In (4.23) all squares are commutative (see Granas and Dugundji [11, p. 377]) and the maps $i_{*}, j_{*}, \widehat{i}_{*}, \widehat{j}_{*}, h_{*}$ are homeomorphisms induced by the corresponding inclusions maps. Moreover, $\partial_{*}$ and $\widehat{\partial}_{*}$ are the corresponding boundary homeomorphisms. Since $\gamma<\xi<\mu_{0}<\inf \widetilde{\varphi}\left(K_{\tilde{\varphi}}\right)$, from the second deformation theorem (see, for example, Gasinski and Papageorgiou [8, p. 628], we know that $\widetilde{\varphi}^{\gamma}$ is a strong deformation retract of $\widetilde{\varphi}^{\xi}$ and so

$$
H_{k}\left(\widetilde{\varphi}^{\xi}, \widetilde{\varphi}^{\gamma}\right)=0 \quad \text { for all } k \geq 0
$$


(see Granas and Dugundji [11, p. 387]). Let $\chi: C_{\rho} \rightarrow C_{R}$ be the map defined by

$$
\chi(u)= \begin{cases}R \frac{u}{\|u\|} & \text { if } \rho \leq\|u\| \leq R, \\ u & \text { if } R<\|u\| .\end{cases}
$$

Evidently $\chi$ is continuous and $\chi_{\mid C_{R}}=\operatorname{id}_{\mid C_{R}}$. So, $C_{R}$ is a retract of $C_{\rho}$. Also, we consider the deformation $h:[0,1] \times C_{\rho} \rightarrow Y$ defined by

$$
h(t, u)=(1-t) u+t R \frac{u}{\|u\|} \text { for all }[t, u] \in[0,1] \times C_{\rho} .
$$

Using $h$, we see that $C_{\rho}$ is deformable into $C_{R}$ over $Y$. Therefore, invoking Theorem 6.5, p. 325 of Dugundji [6], we have that $C_{R}$ is a deformation retract of $C_{\rho}$. This means that

$$
H_{k}\left(C_{\rho}, C_{R}\right)=0 \text { for all } k \geq 0
$$

(see Granas and Dugundji [11, p. 387]). From the exactness of the long homology sequences in (4.23), we have

$$
\begin{array}{llll}
0=\operatorname{im} i_{*}=\operatorname{ker} j_{*} & \text { see }(4.25) & \text { and } & \operatorname{im} j_{*}=\operatorname{ker} \partial_{*}=H_{k}\left(Y, C_{\rho}\right), \\
0=\operatorname{im} \widehat{i}_{*}=\operatorname{ker} \widehat{j}_{*} & \text { see }(4.24) & \text { and } & \operatorname{im} \widehat{j}_{*}=\operatorname{ker} \widehat{\partial}_{*}=H_{k}\left(Y, \widetilde{\varphi}^{\xi}\right) .
\end{array}
$$

It follows that both $j_{*}$ and $\widehat{j}_{*}$ are group isomorphisms and the Lemma D.1, p. 610, of Granas and Dugundji [11] implies that $h_{*}$ is an isomorphism. So, we have

$$
H_{k}\left(Y, C_{\rho}\right)=H_{k}\left(Y, \widetilde{\varphi}^{\xi}\right) \quad \text { for all } k \geq 0 .
$$

Since $\xi<\inf \widetilde{\varphi}\left(K_{\widetilde{\varphi}}\right)$, we obtain

$$
H_{k}\left(Y, C_{\rho}\right)=C_{k}(\widetilde{\varphi}, \infty) \text { for all } k \geq 0 .
$$

Using the radial retraction and Theorem 6.5 of Dugundji [6, p. 325], we show that $\partial B_{\rho}=\{y \in Y:\|y\|=\rho\}$ is a deformation retract of $C_{\rho}$. So

$$
H_{k}\left(Y, C_{\rho}\right)=H_{k}\left(Y, \partial B_{\rho}\right) \quad \text { for all } k \geq 0 .
$$

From Maunder [15, p. 121], we deduce

$$
H_{k}\left(Y, C_{\rho}\right)=\delta_{k, d_{m}} Z, \quad \text { for all } k \geq 0 .
$$

Hence, taking into account (4.26), we obtain

$$
C_{k}(\widetilde{\varphi}, \infty)=\delta_{k, d_{m}} Z \quad \text { for all } k \geq 0 .
$$

Now we introduce the stronger conditions on the reaction $f(z, x)$ :

$H(f)_{3} f: \Omega \times \mathbb{R} \rightarrow \mathbb{R}$ is a measurable function such that for almost all $z \in \Omega$, $f(z, 0)=0, f(z, \cdot) \in C^{1}(\mathbb{R})$ and

(i) there exist integer $m \geq \max \left\{m_{0}, 2\right\}$ and a function $\eta \in L^{\infty}(\Omega)$ such that $\eta(z) \leq \widehat{\lambda}_{m+1}$ almost everywhere in $\Omega, \eta \neq \widehat{\lambda}_{m+1}$ and $\left|f_{x}^{\prime}(z, x)\right| \leq \eta(z)$ for almost all $z \in \Omega$, all $x, y \in \mathbb{R}$; 
(ii) $\widehat{\lambda}_{m} \leq \liminf _{x \rightarrow \pm \infty} f(z, x) / x$ uniformly for almost all $z \in \Omega$;

(iii) there exist $\mu \in(0,2)$ and $\zeta \in L^{\infty}(\Omega), \zeta(z) \leq 0$ almost every in $\Omega$, $\zeta \neq 0$ such that

$\limsup _{x \rightarrow \pm \infty} \frac{f(z, x) x-2 F(z, x)}{|x|^{\mu}} \leq \zeta(z) \quad$ uniformly for a.a. $z \in \Omega$

(iv) $f_{x}^{\prime}(z, 0)=\lim _{x \rightarrow 0} f(z, x) / x \leq \widehat{\lambda}_{1}$ uniformly for almost all $z \in \Omega$ and $f_{x}^{\prime}(\cdot, 0) \neq \widehat{\lambda}_{1}$

Remark 4.9. Hypotheses $H(f)_{3}(\mathrm{i})$, (ii) and the mean value theorem imply that

$\widehat{\lambda}_{m} \leq \liminf _{x \rightarrow \pm \infty} \frac{f(z, x)}{x} \leq \limsup _{x \rightarrow \pm \infty} \frac{f(z, x)}{x} \leq \eta(z) \quad$ uniformly for a.a. $z \in \Omega$.

Similarly, in this case hypothesis $H(f)_{2}(\mathrm{v})$ is automatically satisfied.

THEOREM 4.10. If hypotheses $H(\beta)$ and $H(f)_{3}$ hold, then problem (1.1) has at least fournontrivial solutions

$$
u_{0} \in \operatorname{int} C_{+}, \quad v_{0} \in-\operatorname{int} C_{+} \quad \text { and } \quad y_{0}, \widehat{y} \in C^{1}(\bar{\Omega}) .
$$

Proof. From Theorem 4.7 we already have three nontrivial solutions

$$
u_{0} \in \operatorname{int} C_{+}, \quad v_{0} \in-\operatorname{int} C_{+} \quad \text { and } \quad y_{0} \in C^{1}(\bar{\Omega}) .
$$

Recall that

$$
C_{1}\left(\varphi, u_{0}\right) \neq 0 \text { and } C_{1}\left(\varphi, v_{0}\right) \neq 0
$$

(see (4.18)). Note that $\varphi \in C^{2}\left(H^{1}(\Omega)\right)$ and

$$
\left\langle\varphi^{\prime \prime}\left(u_{0}\right) y, v\right\rangle=\int_{\Omega}(D y, D v)_{\mathbb{R}^{N}} d z+\int_{\Omega} \beta y v d z-\int_{\Omega} f_{x}^{\prime}\left(z, u_{0}\right) y v d z
$$

for all $y, v \in H^{1}(\Omega)$, hence $\varphi^{\prime \prime}\left(u_{0}\right)$ is a Fredholm operator.

By $\sigma\left(\varphi^{\prime \prime}\left(u_{0}\right)\right)$ we denote the spectrum of $\varphi^{\prime \prime}\left(u_{0}\right)$ and assume that $\sigma\left(\varphi^{\prime \prime}\left(u_{0}\right)\right) \subseteq$ $[0,+\infty)$. For $u \in \operatorname{ker}\left(\varphi^{\prime \prime}\left(u_{0}\right)\right)$, we have

$$
-\triangle u(z)=m(z) u(z) \quad \text { a.e. in } \Omega, \quad \frac{\partial u}{\partial n}=0 \quad \text { on } \partial \Omega,
$$

where $m(\cdot)=f_{x}^{\prime}\left(\cdot, u_{0}(\cdot)\right)-\beta(\cdot) \in L^{s}(\Omega)$. Then from (4.28) and Proposition 2.2 of Godoy, Gosseza and Paczka [10] it follows that $\operatorname{dim} \operatorname{ker}\left(\varphi^{\prime \prime}\left(u_{0}\right)\right) \leq 1$ and so we can apply Proposition 2.5 of Bartsch [3] and have

$$
C_{k}\left(\varphi, u_{0}\right)=\delta_{k, 1} Z \text { for all } k \geq 0 .
$$

Similarly we show that

$$
C_{k}\left(\varphi, v_{0}\right)=\delta_{k, 1} Z \quad \text { for all } k \geq 0 .
$$


Recall that, from (4.21) we have

$$
C_{k}\left(\varphi, y_{0}\right)=C_{k}\left(\varphi, \bar{y}_{0}+\xi^{*}\left(\bar{y}_{0}\right)\right)=C_{k}\left(\widetilde{\varphi}, y_{0}\right)=\delta_{k, d_{m}} Z \quad \text { for all } k \geq 0 .
$$

Also, from Proposition 4.8, and Liu and Li [13], we have

$$
C_{k}(\varphi, \infty)=C_{k}(\widetilde{\varphi}, \infty)=\delta_{k, d_{m}} Z \text { for all } k \geq 0 .
$$

Finally from Proposition 3.5, we have

$$
C_{k}(\varphi, 0)=\delta_{k, 0} Z \text { for all } k \geq 0 .
$$

Suppose $K_{\varphi}=\left\{0, u_{0}, v_{0}, y_{0}\right\}$. Then from (4.29)-(4.33) and the Morse relation (see (2.1)) with $t=-1$, we have

$$
(-1)^{0}+2(-1)^{1}+(-1)^{d_{m}}=(-1)^{d_{m}},
$$

that is $-1=0$, a contradiction. So, we can find $\widehat{y} \in K_{\varphi}, \widehat{y} \notin K_{\varphi}=\left\{0, u_{0}, v_{0}, y_{0}\right\}$. Then $\widehat{y}$ is a solution of (1.1) and the regularity result of Wang [21] imply $\widehat{y} \in$ $c^{1}(\bar{\Omega})$.

\section{REFERENCES}

[1] H. Amann, Saddle points and multiple solutions of differential equations, Math. Z. 169 (1979), no. 2, 127-166.

[2] H. Amann And E. Zehnder, Nontrivial solutions for a class of nonresonance problems and applications to nonlinear differential equations, Ann. Scuola Norm. Sup. Pisa Cl. Sci. (4) 7 (1980), no. 4, 539-603.

[3] T. BARTSCH, Critical point theory on partially ordered Hilbert spaces, J. Funct. Anal. 186 (2001), 117-152.

[4] A. Castro And A.C. Lazer, Critical point theory and the number of solutions of a nonlinear Dirichlet problem, Ann. Mat. Pura Appl. (4) 120 (1979), 113-137.

[5] K.C. Chang, S.J. Li AND J.Q. LiU, Remarks on multiple solutions for asymptotically linear elliptic boundary value problems, Topol. Methods Nonlinear Anal. 3 (1994), no. 1, 179-187.

[6] J. Dugundu, Topology, Allyn and Bacon, Boston, Mass. 1966.

[7] M. Filippakis and N.S. PAPAgeorgiou, Multiple nontrivial solutions for resonant Neumann problems, Math. Nachr. 283 (2010), no. 7, 1000-1014.

[8] L. Gasinski and N.S. Papageorgiou, Nonlinear Analysis, Chapman Hall/CRC Press, Boca Raton, FL. (2006).

[9] _ Pairs of nontrivial solutions for resonant Neumann problems, J. Math. Anal. Appl. 398, no. 2, (2013), 649-663.

[10] J. Godoy, J.P. Gossez And S. PaczKa, Antimaximum principle for elliptic problems with weight, Electronic J. Differential Equations 1999, No 22 (1999), 1-15.

[11] A. Granas and J. Dugundj, Fixed Point Theory, Springer-Verlag, New York, 2003.

[12] S. LiU, Remarks on multiple solutions for elliptic resonant problems, J. Math. Anal. Appl. 336 (2007), no. 1, 498-505.

[13] S. LiU And S. Li, Critical groups at infinity, saddle point reduction and elliptic resonant problems, Commun. Contemp. Math. 5 (2003), no. 5, 761-773. 
[14] S. LI AND Z. ZhANG, Multiple solutions theorems for semilinear elliptic boundary value problems with resonance at infinity, Discrete Contin. Dynam. Systems 5 (1999), no. 3, 489-493.

[15] C.R.F. Maunder, Algebraic Topology, Dover Publications, Inc., Mineola, NY (1996).

[16] P. PuCCi AND J. Serrin, The maximum principle, Progress in Nonlinear Differential Equations and their Applications 73, Birkhäuser Verlag, Basel, 2007.

[17] C.L. TANG, Multiple solutions of Neumann problem for elliptic equations, Nonlinear Anal. 54 (2003), no. 4, 637-650.

[18] C.L. TANG AND X.P. WU, Existence and multiplicity for solutions of Neumann problem for semilinear elliptic equations, J. Math. Anal. Appl. 288 (2003), no. 2, 660-670.

[19] K. Thews, Nontrivial solutions of elliptic equations at resonance, Proc. Roy. Soc. Edinburgh Sect. A 85 (1980), no. 1-2, 119-129.

[20] J. VAzQuez, A strong maximum principle for some quasilinear elliptic equations, Appl. Math. Optim. 12 (1984), 191-202.

[21] X.J. WANG, Neumann problems of semilinear elliptic equations involving critical Sobolev exponents, J. Differential Equations 93 (1991), no. 2, 283-310.

Manuscript received March 26, 2013

Giuseppina Barletta And Roberto Livrea

Università degli Studi Mediterranea

di Reggio Calabria

DICEAM

Via Graziella

Località Feo di Vito

89100 Reggio Calabria, ITALY

E-mail address: giuseppina.barletta@unirc.it, roberto.livreaa@unirc.it

Nicolaos S. Papageorgiou

National Technical University

Departement of Mathematics

Zagrafou Campus

Athens 15780, GREECE

E-mail address: npapg@math.ntua.gr

TMNA : VOLUME $45-2015-\mathrm{N}^{\mathrm{O}} 2$ 